\title{
Methane Adsorption in Model Mesoporous Material, SBA-15, Studied by Small-Angle Neutron Scattering
}

Wei-Shan Chiang, ${ }^{\dagger, \ddagger}$ Emiliano Fratini, ${ }^{\perp}$ Piero Baglioni, ${ }^{\perp}$ Daniel Georgi, ${ }^{*}$ Jin-Hong Chen, ${ }^{*}$, and Yun Liu ${ }^{*}$, ,‡

${ }^{\dagger}$ Center for Neutron Research, National Institute of Standards and Technology, Gaithersburg, Maryland, USA

${ }^{\ddagger}$ Department of Chemical and Biomolecular Engineering, University of Delaware, Newark, Delaware, USA

${ }^{\perp}$ Department of Chemistry "Ugo Schiff” and CSGI, University of Florence, via della Lastruccia 3-Sesto Fiorentino, I-50019 Florence, Italy

\#Aramco Services Company: Aramco Research Center-Houston, Texas, USA

\section{Supporting Information}

\section{Scattering Length Density of SBA-15 Matrix}

The scattering length density (SLD) of the SBA-15 matrix is determined by small-angle neutron scattering (SANS) contrast variation method. Five solvents of $\mathrm{D}_{2} \mathrm{O} / \mathrm{H}_{2} \mathrm{O}$ mixture with different $\mathrm{D}_{2} \mathrm{O} / \mathrm{H}_{2} \mathrm{O}$ ratios are prepared to hydrate the SBA-15 powder.

SBA-15 powder is degassed at $120{ }^{\circ} \mathrm{C}$ for 1.5 hours under vacuum before hydrated by the solvents. To hydrate the SBA-15 powder with solvents with correct $\mathrm{D}_{2} \mathrm{O} / \mathrm{H}_{2} \mathrm{O}$ composition, special setup is used to make sure that there are no air bubbles trapped inside small pores of SBA-15 during the hydration. Note that there is small difference in vapor pressure for $\mathrm{D}_{2} \mathrm{O}$ and $\mathrm{H}_{2} \mathrm{O}$ that can shift the $\mathrm{D}_{2} \mathrm{O} / \mathrm{H}_{2} \mathrm{O}$ ratio in a sample if a sample is hydrated by simply exposing it to the $\mathrm{D}_{2} \mathrm{O} / \mathrm{H}_{2} \mathrm{O}$ vapor.

In our setup, SBA-15 powder is loaded into an aluminum bag which is not airtight to allow solvent to flow in but tight enough to keep all the powder inside. The aluminum bag with SBA15 powder is then put in a flask connected by a two-valve vacuum transfer adapter. The adapter is linked to a vacuum pump at one valve (vacuum valve) and a beaker containing a solvent of desired $\mathrm{D}_{2} \mathrm{O} / \mathrm{H}_{2} \mathrm{O}$ composition at the other valve (solvent valve). At the beginning, we vacuum the pre-dried SBA-15 powder in the flask with the vacuum valve open and the solvent valve 
closed. This is to get rid of the air trapped inside the SBA- 15 pores. Then we close the vacuum valve and open the solvent valve. All the solvent is adsorbed into the flask due to the pressure difference. The SBA-15 powder inside the bag is soaked in the solvent with the desired $\mathrm{D}_{2} \mathrm{O} / \mathrm{H}_{2} \mathrm{O}$ composition for half an hour. The whole aluminum bag containing the hydrated SBA15 sample with the desired solvent filling the pores is quickly transferred to an airtight sample cell for SANS measurement.

For all the hydrated samples, we make sure that the degassed dry samples before hydration have the same mass and the neutron beam size is bigger than the aluminum bag to "see" all the hydrated SBA-15 powders inside the sample cell. Therefore, the first order peak intensity after subtracting the background should only depend on the SLD contrast between SBA-15 matrix and the solvent trapped in the pores.

Figure S1a shows the SANS data for SBA-15 samples hydrated with solvents of different $\mathrm{D}_{2} \mathrm{O}$ concentrations. The $1^{\text {st }}$ diffraction peak intensity changes with the $\mathrm{D}_{2} \mathrm{O} / \mathrm{H}_{2} \mathrm{O}$ ratio during the hydration because of the variation of SLD contrast between SBA-15 matrix and the pore solvent $\left(S L D_{\text {matrix }}-S D_{\text {solvent }}\right)$. The peak is subtracted with a linear background and the square root of the background-free peak intensity $\left[I_{\max }(Q)\right]^{0.5}$ as a function of $\mathrm{D}_{2} \mathrm{O}$ mole percentage (mole \%) is plotted in Figure S1b. $\left[I_{\max }(Q)\right]^{0.5}$ is proportional to the SLD contrast between the SBA-15 matrix and pore solvent. For 0 mole $\%$ of $\mathrm{D}_{2} \mathrm{O}$, i.e. $\mathrm{H}_{2} \mathrm{O}$, the SLD contrast is expected to have the reverse sign compared with the 100 mole $\%$ of $\mathrm{D}_{2} \mathrm{O}$ case. Therefore, we add the negative sign to $\left[I_{\max }(Q)\right]^{0.5}$ for the $\mathrm{H}_{2} \mathrm{O}$ hydrated sample. The data can be fitted well, using the linear equation $\left[I_{\max }(Q)\right]^{0.5}=A \times \mathrm{D}_{2} \mathrm{O}$ mole $\%+B$, with the intercept on $\mathrm{D}_{2} \mathrm{O}$ mole $\%$ axis $(B)$ to be 59 $\pm 0.1 \%$, which corresponds to SLD of $(3.51 \pm 0.008) \times 10^{10} \mathrm{~cm}^{-2}$. The confidence intervals for uncertainties represent one standard deviation. This value matches the SBA-15 matrix SLD.

SBA-15 matrix mass density is calculated to be $(2.23 \pm 0.005) \mathrm{g} / \mathrm{cm}^{3}$ by using the relation $\rho_{\text {SLD }}=\frac{\rho_{\text {mass }} N_{A}}{M_{w}} b$, where $\rho_{S L D}$ and $\rho_{\text {mass }}$ are SLD and mass density of SBA-15 solid matrix, respectively. $M_{w}$ is the molecular weight of $\mathrm{SiO}_{2}(60.1=\mathrm{g} / \mathrm{mole}) . b$ is total bound coherent scattering length of one $\mathrm{SiO}_{2}\left(=1.58 \times 10^{-4} \AA\right)$. $N_{A}$ is the Avogadro's constant.

With SLD of SBA-15 matrix ( $\left.\rho_{S B A-15}\right)$ as a known value, we can fit the SANS data of pure SBA15 and $\mathrm{CD}_{4} / \mathrm{SBA}-15$ using equation (1) in the main context: 


$$
\lim _{q_{h, k} L \rightarrow \infty} I\left(q_{h, k}\right)=C \sum_{h, k}\left\{\begin{array}{l}
\frac{1}{q_{h, k}} m_{h, k} \times \\
{\left[\begin{array}{l}
\rho_{a d s} R_{m}^{2} \frac{2 J_{1}\left(q_{h, k} R_{m}\right)}{q_{h, k} R_{m}} \exp \left[-\frac{\left(\sigma q_{h, k}\right)^{2}}{2}\right] \\
-\rho_{a d s} 2 \pi^{2} R_{v}^{2} R_{m}^{2} \times \\
\int_{0}^{2 \pi} d \delta^{\prime} \int_{0}^{\infty} d q_{r}{ }^{\prime} q_{r}{ }^{\prime} \frac{2 J_{1}\left(q_{r}{ }^{\prime} R_{m}\right)}{q_{r} R_{m}} \exp \left[-\frac{\left(\sigma q_{r}^{\prime}\right)^{2}}{2}\right] \frac{2 J_{1}\left(\sqrt{q_{h, k}{ }^{2}+q_{r}{ }^{\prime 2}-2 q_{h, k} q_{r}{ }^{\prime} \cos \left(\delta^{\prime}\right)} R_{v}\right)}{\sqrt{q_{h, k}{ }^{2}+q_{r}{ }^{\prime 2}-2 q_{h, k} q_{r}{ }^{\prime} \cos \left(\delta^{\prime}\right)} R_{v}} \\
-\rho_{S B A-15} R_{m}^{2} \frac{2 J_{1}\left(q_{h, k} R_{m}\right)}{q_{h, k} R_{m}} \exp \left[-\frac{\left(\sigma q_{h, k}\right)^{2}}{2}\right]
\end{array}\right.}
\end{array}\right]^{2}
$$



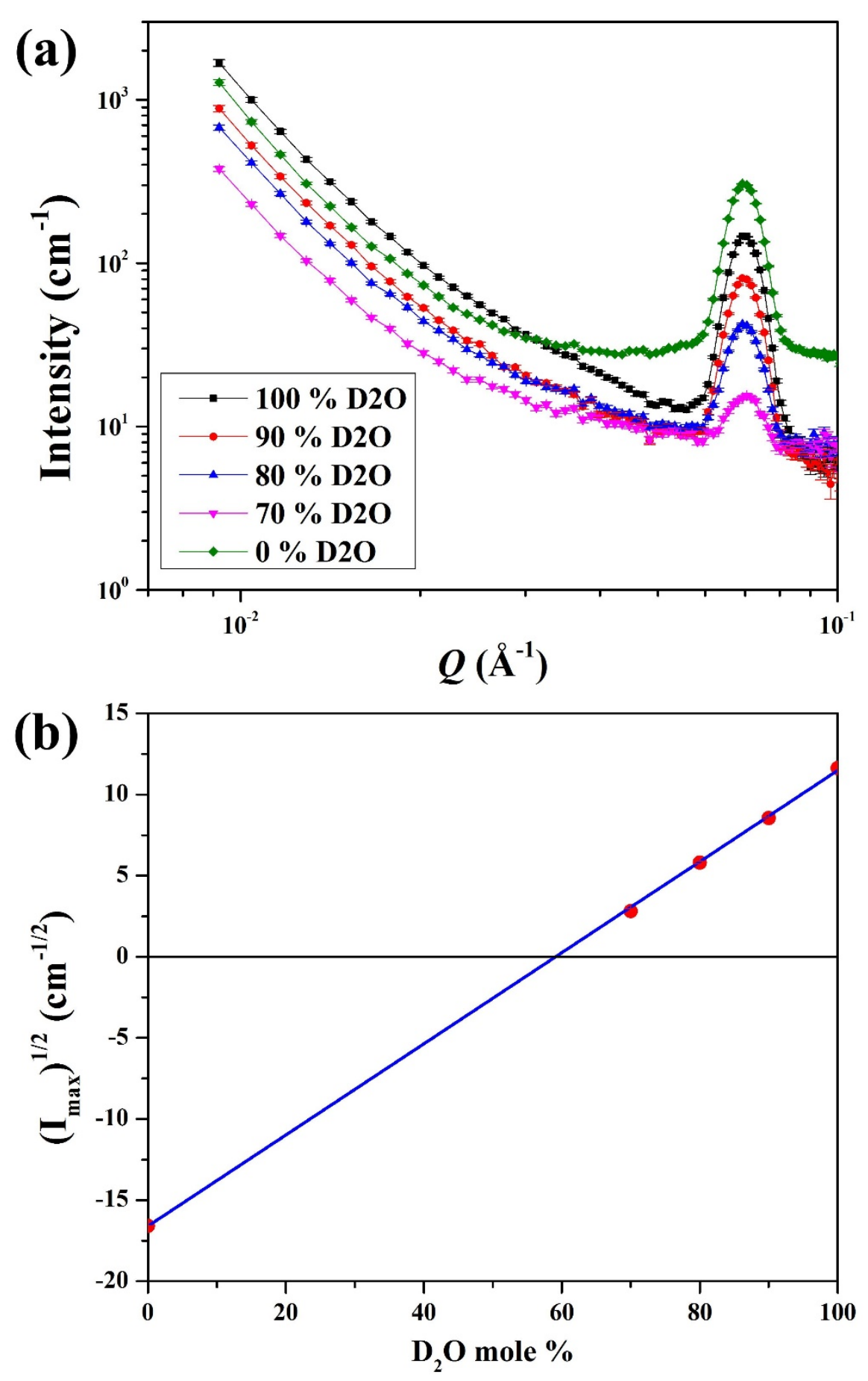

Figure S1. (a) SANS data of SBA-15 powders hydrated with solvents of different $\mathrm{D}_{2} \mathrm{O}$ mole percentages. Error bars represent one standard deviation. (b) The plot of square root of $1^{\text {st }}$ order peak intensity after subtracting the background versus $\mathrm{D}_{2} \mathrm{O}$ mole percentage in the solvent. Error bars represent one standard deviation and are smaller than the symbols. 


\section{Derivation of the Theoretical Model}

Here we show the derivation of equation (1) in the main context in detail.

The coherent scattering intensity of the cylindrical pores of SANS patterns can be expressed as:

$$
I(q)=n\langle P(\vec{q}) S(\vec{q})\rangle
$$

$q$ is the magnitude of scattering vector $\vec{q}$ defined as $q=|\vec{q}|=\frac{4 \pi}{\lambda} \sin \left(\frac{\theta}{2}\right)$, where $\lambda$ and $\theta$ are neutron wave length and scattering angle, respectively. $n$ is a pre-factor related to number density of SBA-15 mesopores. $P(\vec{q})$ is the form factor of SBA-15 cylindrical mesopore with adsorbed and free $\mathrm{CD}_{4}$ confined inside. $S(\vec{q})$ is the structure factor characterizing the hexagonally packed cylindrical mesopores. $<\ldots>$ is the ensemble average over all possible orientations of the SBA15 grains.

Here we neglect the size distribution of the mesopores.

In equation (S1), $P(\vec{q}) \equiv\left|\int \rho(\vec{r}) \exp (i \vec{q} \bullet \vec{r}) d^{3} r\right|^{2}$, where the integration is calculated over the volume of the SBA-15 pore, and $S(\vec{q}) \equiv \frac{1}{N} \sum_{i=1}^{N} \sum_{j=1}^{N} \exp \left(i \vec{q} \cdot\left(\vec{r}_{i}-\vec{r}_{j}\right)\right)$, where $N$ is the number of the mesopores and $r_{i}$ and $r_{j}$ are the position of $i^{\text {th }}$ pore and $j^{\text {th }}$ pore, respectively.

\section{Form Factor}

When $\mathrm{CD}_{4}$ is adsorbed on the surface of SBA-15 cylindrical pores, the pore structure can be treated as core-shell cylinder. The shell is the region where the dense $\mathrm{CD}_{4}$ molecules are adsorbed on the rough surface. It includes the microporous corona region of the solid matrix. The core consists of free $\mathrm{CD}_{4}$ vapor. Here we consider the special case when the interface between SBA-15 matrix and adsorbed $\mathrm{CD}_{4}$ layer is a Gaussian diffusive interface while the interface between adsorbed $\mathrm{CD}_{4}$ layer and $\mathrm{CD}_{4}$ vapor core is a smooth and sharp interface. We define $R_{v}$ as the radius of $\mathrm{CD}_{4}$ vapor core, $R_{m}$ as the nominal radius of the mesopore, and $L$ is the length of the cylindrical pore. $\rho_{v}, \rho_{a d s}$, and $\rho_{S B A-15}$ are scattering length density (SLD) of the $\mathrm{CD}_{4}$ vapor core, adsorbed $\mathrm{CD}_{4}$ shell, and SBA-15 matrix, respectively. $\sigma$ is the diffusive parameter used to characterize the roughness of the SBA- 15 pore surface. Since the free vapor $\mathrm{CD}_{4}$ has density much smaller than the density of adsorbed $\mathrm{CD}_{4}$ in the pressure and temperature conditions being studied here, we assume $\rho_{v}=0$. 
To derive the equation of form factor for a cylindrical mesopore with gas adsorption on the rough surface, the following terms are defined as:

$$
\begin{aligned}
& \text { Filled Pore Term } \equiv \frac{\sin (q L \cos \alpha / 2)}{(q L \cos \alpha / 2)} \rho_{a d s} V_{m} \frac{2 J_{1}\left(q R_{m} \sin \alpha\right)}{q R_{m} \sin \alpha} \exp \left[-\frac{(\sigma q \sin \alpha)^{2}}{2}\right] \\
& \text { Filled Core Term } \equiv \frac{\sin (q L \cos \alpha / 2)}{(q L \cos \alpha / 2)} \rho_{a d s} V_{v} \frac{2 J_{1}\left(q R_{v} \sin \alpha\right)}{q R_{v} \sin \alpha}
\end{aligned}
$$

Core - Shell Pore Term $=$ Filled Pore Term - Filled CoreTerm

$\alpha$ is the angle between cylinder axis and wave vector $\vec{q}, J_{1}$ is the first order Bessel function, $V_{m}=\pi R_{m}^{2} L$ and $V_{v}=\pi R_{v}^{2} L$. Filled Pore Term represents the form factor of a cylinder of radius $R_{m}$ with Gaussian diffusive interface and with the cylinder fully filled by pore fluid with SLD of $\rho_{\text {ads }}$. Filled Core Term is the form factor of a cylinder of radius $R_{v}$ with sharp interface and with the cylinder fully filled by pore fluid with SLD of $\rho_{\text {ads }}$.

A Modified Filled Core Term in "real space" is calculated as Filled Pore Term in "real space" multiplying a truncation function $T(r)$ :

Modified Filled Core Term $(r)=$ Filled Pore Term $(r) \times T(r)$,

where

$T\left(r, R_{v}\right)=1 \quad$ if $r \leq R_{v}$

$T\left(r, R_{v}\right)=0 \quad$ if $r>R_{v}$

$T\left(r, R_{i n}\right)$ in reciprocal space, i.e. $T\left(q, R_{i n}\right)$, is just a cylinder form factor with cylinder radius $R=$ $R_{V}$.

Therefore, we can calculate the Modified Filled Core Term in reciprocal space as the form factor of SBA-15 mesopore with Gaussian diffuse interface fully filled by $\mathrm{CD}_{4}$ with SLD of $\rho_{\text {ads }}$ (Filled Pore Term described in equation (S2)) convoluted with the form factor of cylinder of radius $R_{v}$ with sharp interface. 
Modified Filled CoreTerm =

$$
\begin{aligned}
& \left\{\frac{\sin (q L \cos \alpha / 2)}{(q L \cos \alpha / 2)} \rho_{a d s} V_{m} \frac{2 J_{1}\left(q R_{m} \sin \alpha\right)}{q R_{m} \sin \alpha} \exp \left[-\frac{(\sigma q \sin \alpha)^{2}}{2}\right]\right\} \otimes \\
& \left\{\frac{\sin (q L \cos \alpha / 2)}{(q L \cos \alpha / 2)} V_{v} \frac{2 J_{1}\left(q R_{v} \sin \alpha\right)}{q R_{v} \sin \alpha}\right\} \\
& =\left\{\frac{\sin \left(q_{z} L / 2\right)}{\left(q_{z} L / 2\right)} \rho_{a d s} V_{m} \frac{2 J_{1}\left(q_{r} R_{m}\right)}{q_{r} R_{m}} \exp \left[-\frac{\left(\sigma q_{r}\right)^{2}}{2}\right]\right\} \otimes\left\{\frac{\sin \left(q_{z} L / 2\right)}{\left(q_{z} L / 2\right)} V_{v} \frac{2 J_{1}\left(q_{r} R_{v}\right)}{q_{r} R_{v}}\right\} \\
& =\rho_{a d s} V_{m} V_{v} \int_{-\infty}^{\infty} \frac{\sin \left(q_{z}^{\prime} L / 2\right)}{\left(q_{z}^{\prime} L / 2\right)} \frac{\sin \left(\left(q_{z}-q_{z}{ }^{\prime}\right) L / 2\right)}{\left(\left(q_{z}-q_{z}{ }^{\prime}\right) L / 2\right)} d q_{z}{ }^{\prime} \times
\end{aligned}
$$

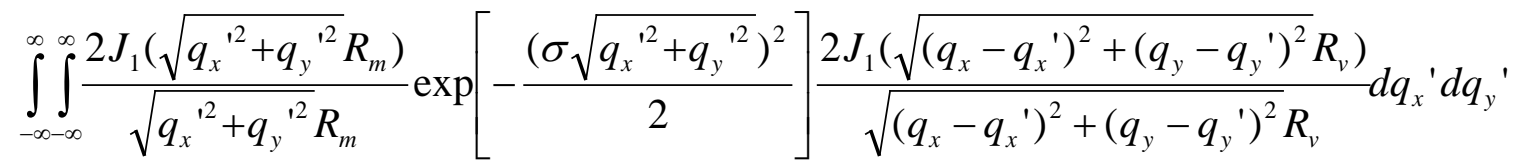

$$
\begin{aligned}
& =\rho_{a d s} V_{m} V_{v} \int_{-\infty}^{\infty} \frac{\sin \left(q_{z}{ }^{\prime} L / 2\right)}{\left(q_{z}{ }^{\prime} L / 2\right)} \frac{\sin \left(\left(q_{z}-q_{z}{ }^{\prime}\right) L / 2\right)}{\left(\left(q_{z}-q_{z}{ }^{\prime}\right) L / 2\right)} d q_{z}{ }^{\prime} \times \\
& \int_{0}^{2 \pi} d \delta^{\prime} \int_{0}^{\infty} d q_{r}{ }^{\prime} q_{r}{ }^{\prime} \frac{2 J_{1}\left(q_{r}{ }^{\prime} R_{m}\right)}{q_{r}{ }^{\prime} R_{m}} \exp \left[-\frac{\left(\sigma q_{r}{ }^{\prime}\right)^{2}}{2}\right] \frac{2 J_{1}\left(\sqrt{q_{r}{ }^{2}+q_{r}{ }^{\prime 2}-2 q_{r} q_{r}{ }^{\prime} \cos \left(\delta^{\prime}\right)} R_{v}\right)}{\sqrt{q_{r}{ }^{2}+q_{r}{ }^{\prime 2}-2 q_{r} q_{r}{ }^{\prime} \cos \left(\delta^{\prime}\right)} R_{v}}
\end{aligned}
$$

Since $\int_{-\infty}^{\infty} \frac{\sin \left(q_{z}{ }^{\prime} L / 2\right)}{\left(q_{z}{ }^{\prime} L / 2\right)} \frac{\sin \left(\left(q_{z}-q_{z}{ }^{\prime}\right) L / 2\right)}{\left(\left(q_{z}-q_{z}{ }^{\prime}\right) L / 2\right)} d q_{z}{ }^{\prime}=\frac{2 \pi}{L} \frac{\sin \left(q_{z} L / 2\right)}{\left(q_{z} L / 2\right)}$, then

$$
\begin{aligned}
& \text { Modified Filled Core Term }=\rho_{\text {ads }} V_{m} V_{v} \frac{2 \pi}{L} \frac{\sin \left(q_{z} L / 2\right)}{\left(q_{z} L / 2\right)} \times \\
& \int_{0}^{2 \pi} d \delta^{\prime} \int_{0}^{\infty} d q_{r}{ }^{\prime} q_{r}{ }^{\prime} \frac{2 J_{1}\left(q_{r}{ }^{\prime} R_{m}\right)}{q_{r}{ }^{\prime} R_{m}} \exp \left[-\frac{\left(\sigma q_{r}\right)^{2}}{2}\right] \frac{2 J_{1}\left(\sqrt{q_{r}{ }^{2}+q_{r}{ }^{2}-2 q_{r} q_{r}{ }^{\prime} \cos \left(\delta^{\prime}\right)} R_{v}\right)}{\sqrt{q_{r}{ }^{2}+q_{r}{ }^{2}-2 q_{r} q_{r}{ }^{\prime} \cos \left(\delta^{\prime}\right)} R_{v}}
\end{aligned}
$$

Replace $\frac{\sin (q L \cos \alpha / 2)}{(q L \cos \alpha / 2)} \rho_{a d s} V_{v} \frac{2 J_{1}\left(q R_{v} \sin \alpha\right)}{q R_{v} \sin \alpha}$ in equation (S3) (the second term) by Modified Filled Core Term, we obtain: 


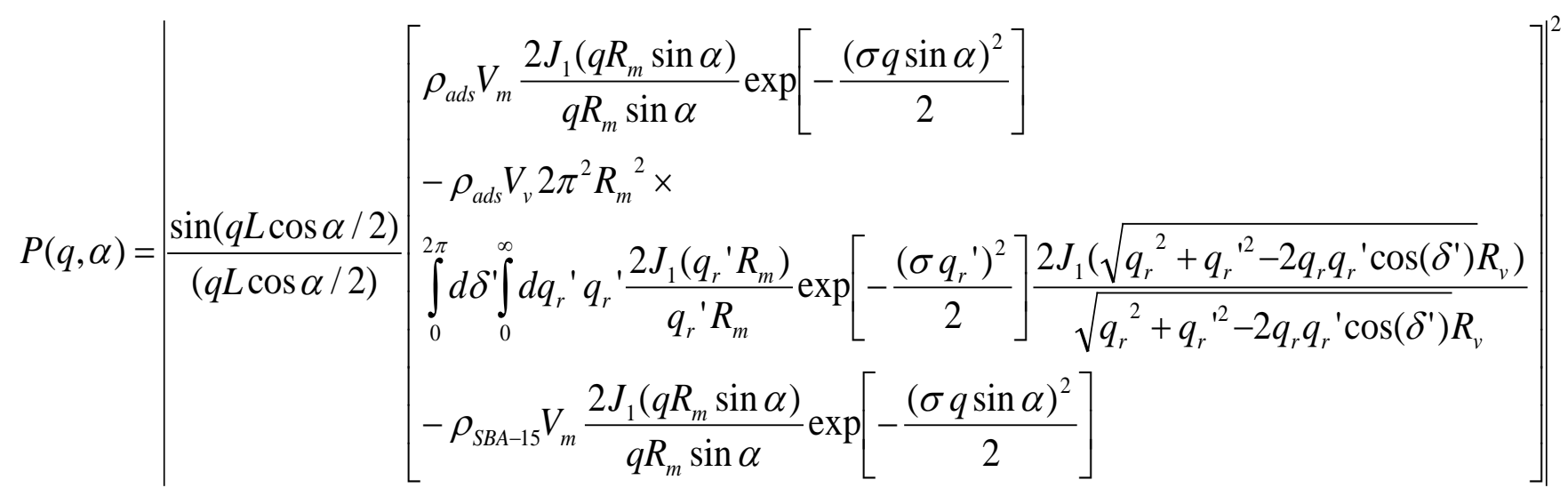

Issue of Previous Model for Form Factor

In previous literature, Muroyama et al. (reference [13] in the main context) has used similar model with Gaussian diffuse interfaces for the Argon adsorption on the MCM-41 mesoporous material. MCM-41 has hexagonally packed cylindrical mesopores similar to the structure of SBA-15. However, in their model, both the interface between the matrix and the adsorbed layer $\left(I n_{m-a d s}\right)$ and the interface between the adsorbed layer and the free vapor core $\left(I n_{a d s-v}\right)$ are assumed to be Gaussian diffuse interfaces and have the same diffusive parameter $\sigma$. The corresponding terms in the paper of Muroyama et al. for Filled Pore Term $(M)$, negative Filled ${\text { Core } \operatorname{Term}_{(M)} \text {, and Core-Shell Pore Term }}_{(M)}$ in reciprocal space with $\alpha=90^{\circ}$ are:

Filled Pore $\operatorname{Term}_{(M)} \equiv \rho_{a d s} V_{m} \frac{2 J_{1}\left(q R_{m}\right)}{q R_{m}} \exp \left[-\frac{(\sigma q)^{2}}{2}\right]$

Filled Core $\operatorname{Term}_{(M)} \equiv \rho_{a d s} V_{v} \frac{2 J_{1}\left(q R_{v}\right)}{q R_{v}} \exp \left[-\frac{(\sigma q)^{2}}{2}\right]$

Core-Shell Pore Term $_{(M)}=$ Filled Pore Term $(M)-$ Filled Core Term $(M)$

The radial distribution of the Filled Pore $\operatorname{Term}_{(M)}$, negative Filled Core Term $(M)$, and Core-Shell

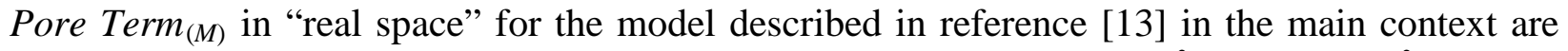
plotted in Figure S2a. The parameters used for Figure S2 are $R_{v}=38.0 \AA, R_{m}=34.1 \AA, \sigma=7.5$ $\AA$, and $\alpha=90^{\circ}$ and they are in the category of $R_{v}>R_{m}$. The Core-Shell Pore Term $(M)$ represents the volume average SLD of adsorbed gas layer and it is proportional to volume average mass density of gas molecules in the dense adsorption region. When $R_{v}>R_{m}$, it is shown clearly in Figure S2a that the model described by Muroyama et al. ends up with negative SLD and mass 
density of gas molecules and this is physically unreasonable. Therefore, it should be noticed that the model proposed in reference [13] in the main context should be used with caution and it is only valid when $R_{v}<R_{m}$.

The new equation derived here with Modified Filled Core Term (included in equation (S6)) has fixed this unphysical problem of negative mass density of dense gas molecules. Here the interface $I n_{m \text {-ads }}$ is assumed to be Gaussian diffuse interface and $I n_{a d s-v}$ is assumed to be sharp and smooth interface. It is worth nothing that in equation (S6), $R_{v}$ can be larger than $R_{m}$, which is very different than the regular core-shell model with sharp interfaces.

Figure S2b demonstrates the corresponding radial distribution of Modified Filled Pore Term (= Filled Pore Term), Modified Filled Core Term, and Modified Core-Shell Pore Term in real space when the Filled Core Term in equation (S3) is modified to correct the negative mass density real space (equation (S6)). The parameters are the same as what used in Figure S2a. 
(a)

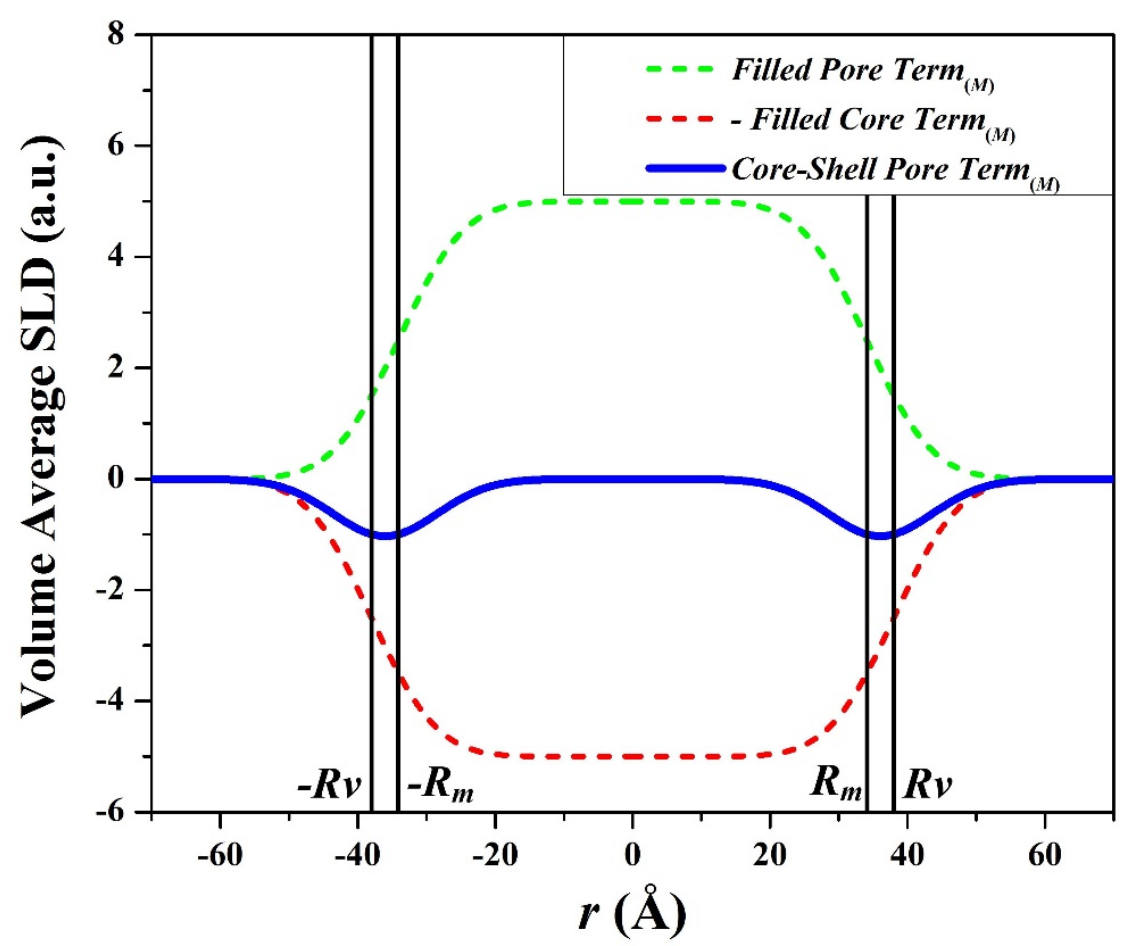

(b)

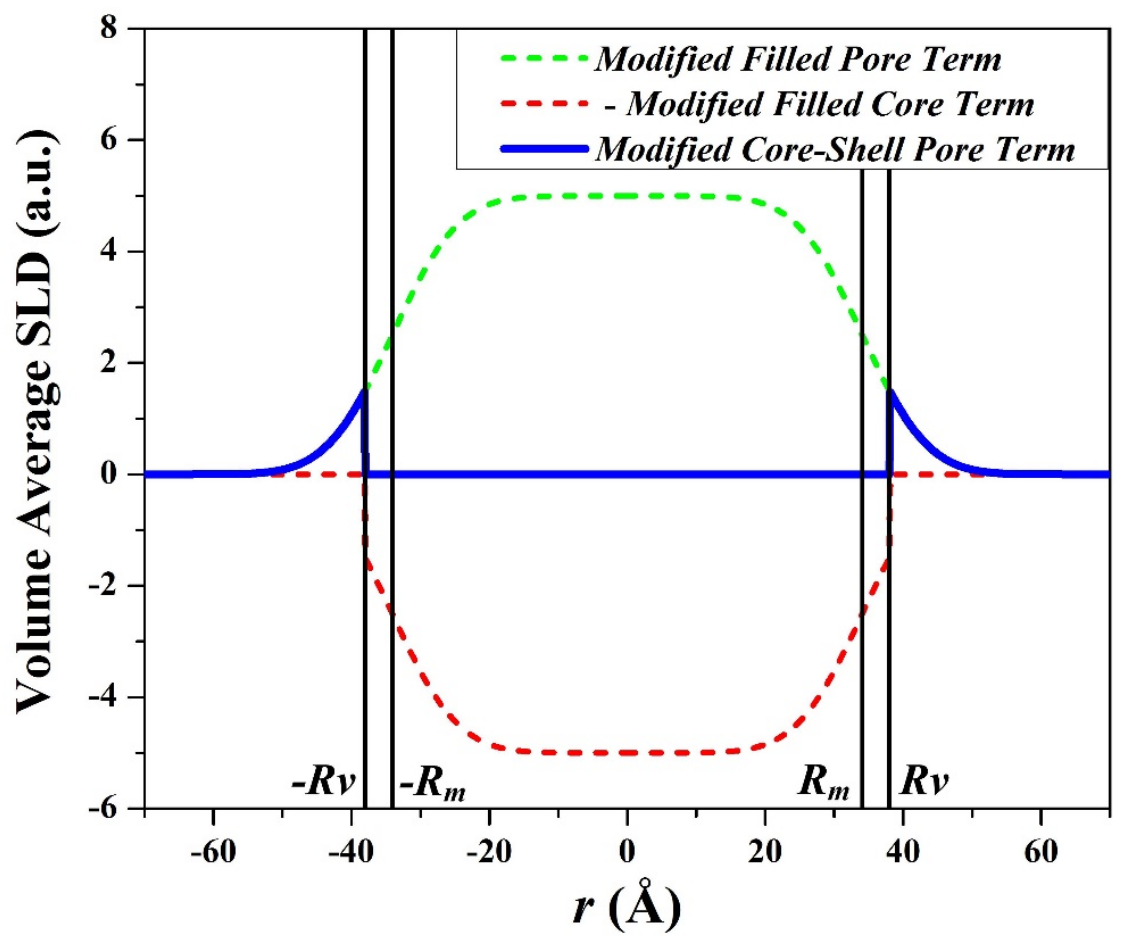

Figure S2. Radial distribution of Filled Pore Term (green dash line), negative Filled Core Term (red dash line), and Core-Shell Pore Term (blue solid line) in "real space" when (a) using the model of Muroyama et al. described in reference [13] of the main context (equation (S7)-(S9)) and (b) using our model (equation (S6)). 
For hexagonally packed cylindrical core-shell pores, the structure factor $S(\vec{q})$ is the summation of the delta functions located at hexagonal lattice points in reciprocal space $\overrightarrow{q_{h, k}}$.

$$
\begin{aligned}
& I(q)=\frac{1}{4 \pi} \int_{\Omega} P(\vec{q}, \alpha) S(\vec{q}) d \Omega=\frac{1}{4 \pi} \int_{\Omega} P(\vec{q}, \alpha) \sum_{h, k} \delta\left(\overrightarrow{q_{x, y}}-\overrightarrow{q_{h, k}}\right) d \Omega \\
& =\frac{1}{4 \pi} \int_{0}^{2 \pi} d \phi \int_{0}^{\pi} \sin \alpha d \alpha P(\vec{q}, \alpha) \sum_{h, k} \delta\left(\overrightarrow{q_{x, y}}-\overrightarrow{q_{h, k}}\right) \\
& =\frac{1}{4 \pi} \int_{0}^{2 \pi} d \phi \int_{0}^{\pi} \sin \alpha d \alpha P(\vec{q}, \alpha) \sum_{h, k} \delta\left(\left|q_{x, y}\right|-\left|q_{h, k}\right|\right) \delta\left(\phi-\phi_{h, k}\right) \\
& =\frac{1}{4 \pi} \sum_{h, k} \int_{0}^{2 \pi} d \phi \delta\left(\phi-\phi_{h, k}\right) \int_{0}^{\pi} \sin \alpha P(\vec{q}, \alpha) \delta\left(\left|q_{x, y}\right|-\left|q_{h, k}\right|\right) d \alpha \\
& =\frac{1}{4 \pi} \sum_{h, k} \int_{0}^{2 \pi} d \phi \delta\left(\phi-\phi_{h, k}\right) \times
\end{aligned}
$$

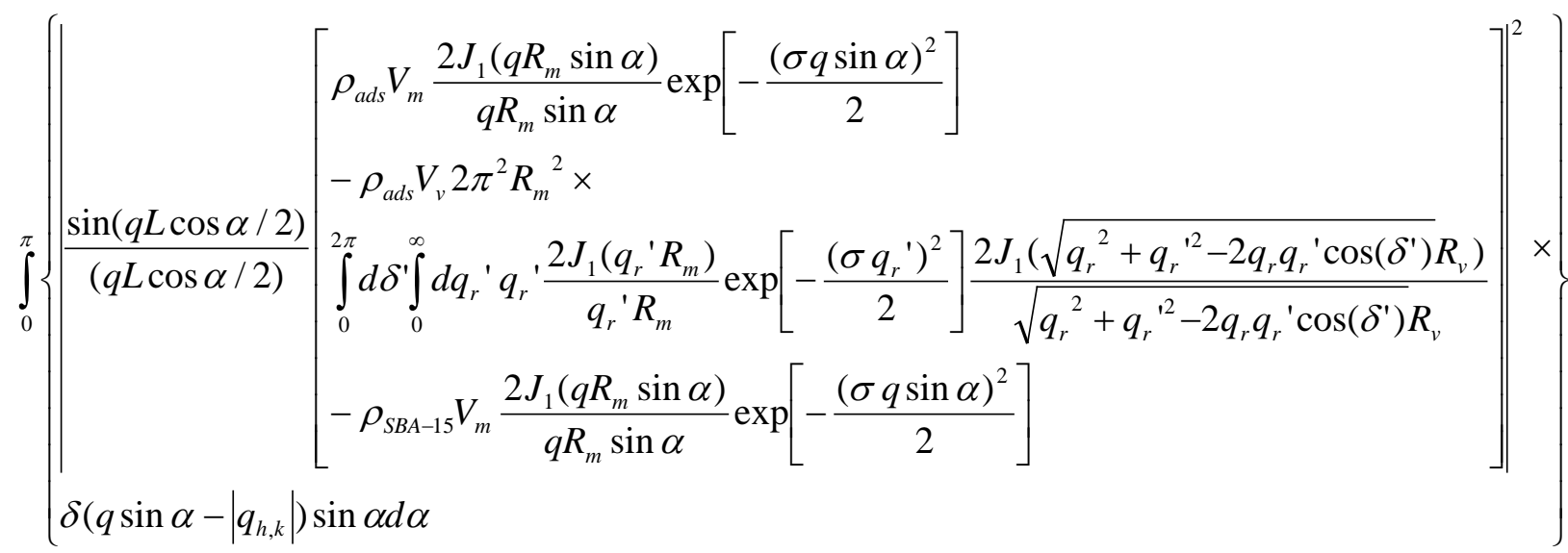


Define $\mu=\cos \alpha$, then

$$
\left.\begin{array}{l}
I(q)=\frac{1}{4 \pi} \sum_{h, k}^{2 \pi} \int_{0}^{2 \pi} d \phi \delta\left(\phi-\phi_{h, k}\right) \times \\
\left\{\begin{array}{l}
\left(\frac{\sin (q L \mu / 2)}{(q L \mu / 2)}\right)^{2} \times \\
{\left[\begin{array}{l}
\rho_{a d s} V_{m} \frac{2 J_{1}\left(q R_{m} \sqrt{1-\mu^{2}}\right)}{q R_{m} \sqrt{1-\mu^{2}}} \exp \left[-\frac{\left(\sigma q \sqrt{1-\mu^{2}}\right)^{2}}{2}\right] \\
-\rho_{a d s} V_{v} 2 \pi^{2} R_{m}{ }^{2} \times
\end{array}\right.} \\
\int_{-1}^{2 \pi} \int_{0}^{\infty} d \delta^{\prime} \int_{0}^{\infty} d q_{r}{ }^{\prime} q_{r}{ }^{\prime} \frac{2 J_{1}\left(q_{r}{ }^{\prime} R_{m}\right)}{q_{r} R_{m}} \exp \left[-\frac{\left(\sigma q_{r}{ }^{\prime}\right)^{2}}{2}\right] \frac{2 J_{1}\left(\sqrt{q^{2}\left(1-\mu^{2}\right)+q_{r}{ }^{\prime 2}-2 q \sqrt{1-\mu^{2}} q_{r}{ }^{\prime} \cos \left(\delta^{\prime}\right)} R_{v}\right)}{\sqrt{q^{2}\left(1-\mu^{2}\right)+q_{r}{ }^{\prime 2}-2 q \sqrt{1-\mu^{2}} q_{r}{ }^{\prime} \cos \left(\delta^{\prime}\right) R_{v}}} \\
-\rho_{S B A-15} V_{m} \frac{2 J_{1}\left(q R_{m} \sqrt{1-\mu^{2}}\right)}{q R_{m} \sqrt{1-\mu^{2}}} \exp \left[-\frac{\left(\sigma q \sqrt{1-\mu^{2}}\right)^{2}}{2}\right] \\
\delta\left(q \sqrt{1-\mu^{2}}-\left|q_{h, k}\right|\right) d \mu
\end{array}\right]
\end{array}\right\}
$$

Let $x=q L \mu / 2, \quad \mu=\frac{2 x}{q L}$ 


$$
\begin{aligned}
& I(q)=\frac{1}{4 \pi} \sum_{h, k} \int_{0}^{2 \pi} d \phi \delta\left(\phi-\phi_{h, k}\right) \times \\
& \int_{-q L / 2}^{q L / 2}\left(\frac{2}{q L}\right)\left(\frac{\sin (x)}{(x)}\right)^{2} \times \\
& \int\left[\rho_{a d s} V_{m} \frac{2 J_{1}\left(q R_{m} \sqrt{1-\left(\frac{2 x}{q L}\right)^{2}}\right)}{q R_{m} \sqrt{1-\left(\frac{2 x}{q L}\right)^{2}}} \exp \left[-\frac{\left(\sigma q \sqrt{1-\left(\frac{2 x}{q L}\right)^{2}}\right)^{2}}{2}\right]\right. \\
& -\rho_{a d s} V_{v} 2 \pi^{2} R_{m}^{2} \times \\
& \left\{\int_{0}^{2 \pi} d \delta^{\prime} \int_{0}^{\infty} d q_{r}{ }^{\prime} q_{r}{ }^{\prime} \frac{2 J_{1}\left(q_{r}{ }^{\prime} R_{m}\right)}{q_{r}{ }^{\prime} R_{m}} \exp \left[-\frac{\left(\sigma q_{r}{ }^{\prime}\right)^{2}}{2}\right] \frac{2 J_{1}\left(\sqrt{q^{2}\left(1-\left(\frac{2 x}{q L}\right)^{2}\right)+q_{r}{ }^{\prime 2}-2 q \sqrt{1-\left(\frac{2 x}{q L}\right)^{2}} q_{r}{ }^{\prime} \cos \left(\delta^{\prime}\right) R_{v}}\right)}{\sqrt{q^{2}\left(1-\left(\frac{2 x}{q L}\right)^{2}\right)+q_{r}{ }^{\prime 2}-2 q \sqrt{1-\left(\frac{2 x}{q L}\right)^{2}} q_{r}{ }^{\prime} \cos \left(\delta^{\prime}\right) R_{v}}}\right. \\
& -\rho_{\text {SBA-15 }} V_{m} \frac{2 J_{1}\left(q R_{m} \sqrt{1-\left(\frac{2 x}{q L}\right)^{2}}\right)}{q R_{m} \sqrt{1-\left(\frac{2 x}{q L}\right)^{2}}} \exp \left[-\frac{\left(\sigma q \sqrt{1-\left(\frac{2 x}{q L}\right)^{2}}\right)^{2}}{2}\right] \\
& \delta\left(q \sqrt{1-\left(\frac{2 x}{q L}\right)^{2}}-\left|q_{h, k}\right|\right) d x
\end{aligned}
$$

When $q L \rightarrow \infty, \sqrt{1-\left(\frac{2 x}{q L}\right)^{2}}$ decays slowly from 1 with $x$. 


$$
\begin{aligned}
& \lim _{q L \rightarrow \infty} I(q)=\frac{1}{4 \pi} \sum_{h, k} \int_{0}^{2 \pi} d \phi \delta\left(\phi-\phi_{h, k}\right) \int_{-q L / 2}^{q L / 2}\left(\frac{2}{q L}\right)\left(\frac{\sin (x)}{(x)}\right)^{2} \times \\
& {\left[\begin{array}{l}
\rho_{a d s} V_{m} \frac{2 J_{1}\left(q R_{m}\right)}{q R_{m}} \exp \left[-\frac{(\sigma q)^{2}}{2}\right] \\
-\rho_{a d s} V_{v} 2 \pi^{2} R_{m}{ }^{2} \times \\
\int_{0}^{2 \pi} d \delta^{\prime} \int_{0}^{\infty} d q_{r} q_{r} \frac{2 J_{1}\left(q_{r}{ }^{\prime} R_{m}\right)}{q_{r} R_{m}} \exp \left[-\frac{\left(\sigma q_{r}\right)^{2}}{2}\right] \frac{2 J_{1}\left(\sqrt{q^{2}+q_{r}{ }^{2}-2 q q_{r}{ }^{\prime} \cos \left(\delta^{\prime}\right)} R_{v}\right)}{\sqrt{q^{2}+q_{r}{ }^{\prime 2}-2 q q_{r}{ }^{\prime} \cos \left(\delta^{\prime}\right) R_{v}}} \\
-\rho_{\text {SBA-15 }} V_{m} \frac{2 J_{1}\left(q R_{m}\right)}{q R_{m}} \exp \left[-\frac{(\sigma q)^{2}}{2}\right]
\end{array}\right]^{2}} \\
& \delta\left(q-\left|q_{h, k}\right|\right) d x
\end{aligned}
$$

The $\delta\left(q-\left|q_{h, k}\right|\right)$ term indicates that there is only intensity when $q=\left|q_{h, k}\right|$.

Therefore,

$$
\lim _{q_{h, L} L \rightarrow \infty} I\left(q_{h, k}\right)=\sum_{h, k}\left\{\begin{array}{l}
\frac{1}{4 \pi}\left(\frac{2}{q_{h, k} L}\right) \times \\
{\left[\begin{array}{l}
\rho_{a d s} V_{m} \frac{2 J_{1}\left(q_{h, k} R_{m}\right)}{q_{h, k} R_{m}} \exp \left[-\frac{\left(\sigma q_{h, k}\right)^{2}}{2}\right] \\
-\rho_{a d s} V_{v} 2 \pi^{2} R_{m}{ }^{2} \times \\
\int_{0}^{2 \pi} d \delta^{\prime} \int_{0}^{\infty} d q_{r}{ }^{\prime} q_{r}{ }^{\prime} \frac{2 J_{1}\left(q_{r}{ }^{\prime} R_{m}\right)}{q_{r}{ }^{\prime} R_{m}} \exp \left[-\frac{\left(\sigma q_{r}{ }^{\prime}\right)^{2}}{2}\right] \frac{2 J_{1}\left(\sqrt{q_{h, k}{ }^{2}+q_{r}{ }^{\prime 2}-2 q_{h, k} q_{r}{ }^{\prime} \cos \left(\delta^{\prime}\right)}\right.}{\sqrt{q_{h, k}{ }^{2}+q_{r}{ }^{\prime 2}-2 q_{h, k} q_{r}{ }^{\prime} \cos \left(\delta^{\prime}\right)} R_{v}} \\
-\rho_{S B A-15} V_{m} \frac{2 J_{1}\left(q_{h, k} R_{m}\right)}{q_{h, k} R_{m}} \exp \left[-\frac{\left(\sigma q_{h, k}\right)^{2}}{2}\right] \\
\left(\frac{\sin \left(x_{h, k}\right)}{\left(x_{h, k}\right)}\right)^{2} \int_{0}^{2 \pi} d \phi \delta\left(\phi-\phi_{h, k}\right)
\end{array}\right.}
\end{array}\right\}
$$

Let $\left(\frac{\sin \left(x_{h, k}\right)}{\left(x_{h, k}\right)}\right)^{2}=C_{1}=$ const and

$\int_{0}^{2 \pi} d \phi \delta\left(\phi-\phi_{h, k}\right)=m_{h, k}$, where $m_{h, k}$ is the multiplicity. 


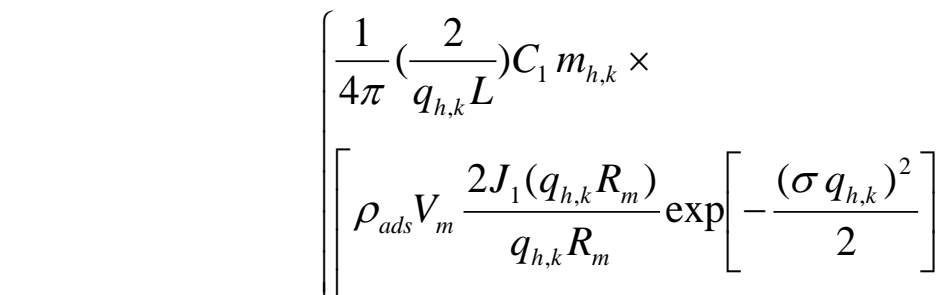

$$
\begin{aligned}
& \lim _{q_{h, k} L \rightarrow \infty} I\left(q_{h, k}\right)=\sum_{h, k}\left\{\begin{array}{l}
-\rho_{a d s} V_{v} 2 \pi^{2} R_{m}{ }^{2} \times \\
\int_{0}^{2 \pi} d \delta^{\prime} \int_{0}^{\infty} d q_{r}{ }^{\prime} q_{r}{ }^{\prime} \frac{2 J_{1}\left(q_{r}{ }^{\prime} R_{m}\right)}{q_{r}{ }^{\prime} R_{m}} \exp \left[-\frac{\left(\sigma q_{r}{ }^{\prime}\right)^{2}}{2}\right. \\
-\rho_{\text {SBA-15 }} V_{m} \frac{2 J_{1}\left(q_{h, k} R_{m}\right)}{q_{h, k} R_{m}} \exp \left[-\frac{\left(\sigma q_{h, k}\right)^{2}}{2}\right]
\end{array}\right. \\
& =C_{2} \sum_{h, k} \frac{1}{q_{h, k}} m_{h, k}\left[\begin{array}{l}
\rho_{a d s} V_{m} \frac{2 J_{1}\left(q_{h, k} R_{m}\right)}{q_{h, k} R_{m}} \exp \left[-\frac{\left(\sigma q_{h, k}\right)^{2}}{2}\right] \\
-\rho_{a d s} V_{v} 2 \pi^{2} R_{m}{ }^{2} \times \\
\int_{0}^{2 \pi} d \delta^{\prime} \int_{0}^{\infty} d q_{r}{ }^{\prime} q_{r}{ }^{\prime} \frac{2 J_{1}\left(q_{r}{ }^{\prime} R_{m}\right)}{q_{r}{ }^{\prime} R_{m}} \exp \left[-\frac{\left(\sigma q_{r}{ }^{\prime}\right)^{2}}{2}\right] \frac{2 J_{1}\left(\sqrt{q_{h, k}{ }^{2}+q_{r}{ }^{\prime 2}-2 q_{h, k} q_{r}{ }^{\prime} \cos \left(\delta^{\prime}\right)} R_{v}\right)}{\sqrt{q_{h, k}{ }^{2}+q_{r}{ }^{\prime 2}-2 q_{h, k} q_{r}{ }^{\prime} \cos \left(\delta^{\prime}\right)} R_{v}} \\
-\rho_{\text {SBA-15 }} V_{m} \frac{2 J_{1}\left(q_{h, k} R_{m}\right)}{q_{h, k} R_{m}} \exp \left[-\frac{\left(\sigma q_{h, k}\right)^{2}}{2}\right]
\end{array}\right]^{2}
\end{aligned}
$$

, where $C_{2}=\frac{1}{4 \pi} \frac{2}{L} C_{1}$ is a constant 


$$
\begin{aligned}
& \left\{\begin{array}{l}
\frac{1}{q_{h, k}} m_{h, k} \times \\
{\left[\begin{array}{l}
\rho_{a d s} \frac{2 J_{1}\left(q_{h, k} R_{m}\right)}{q_{h, k} R_{m}} \exp \left[-\frac{\left(\sigma q_{h, k}\right)^{2}}{2}\right]
\end{array}\right.}
\end{array}\right. \\
& \lim _{q_{h, k} L \rightarrow \infty} I\left(q_{h, k}\right)=C_{2} V_{m}^{2} \sum_{h, k}\left\{-\rho_{a d s} \frac{V_{v}}{V_{m}} 2 \pi^{2} R_{m}^{2} \times\right.
\end{aligned}
$$

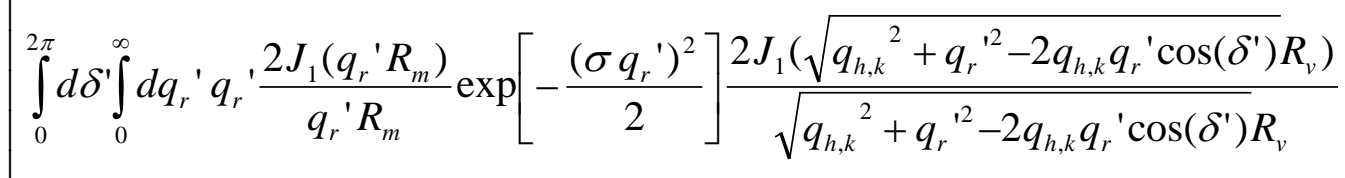

$$
\begin{aligned}
& {\left[-\rho_{\text {SBA- } 15} \frac{2 J_{1}\left(q_{h, k} R_{m}\right)}{q_{h, k} R_{m}} \exp \left[-\frac{\left(\sigma q_{h, k}\right)^{2}}{2}\right]\right.} \\
& \left\{\begin{array}{l}
\frac{1}{q_{h, k}} m_{h, k} \times \\
{\left[\rho_{a d s} \frac{2 J_{1}\left(q_{h, k} R_{m}\right)}{q_{h, k} R_{m}} \exp \left[-\frac{\left(\sigma q_{h, k}\right)^{2}}{2}\right]\right.}
\end{array}\right. \\
& =C_{2}\left(\pi R_{m}^{2} L\right)^{2} \sum_{h, k}\left\{\begin{array}{l}
-\rho_{a d s} 2 \pi^{2} R_{v}^{2} \times \\
2 \pi \quad \infty
\end{array}\right.
\end{aligned}
$$

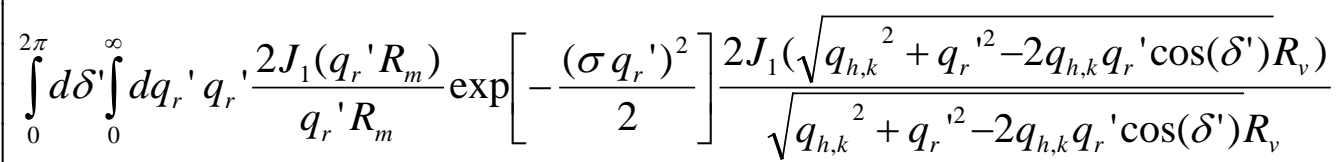

$$
\begin{aligned}
& -\rho_{\text {SBA-15 }} \frac{2 J_{1}\left(q_{h, k} R_{m}\right)}{q_{h, k} R_{m}} \exp \left[-\frac{\left(\sigma q_{h, k}\right)^{2}}{2}\right] \\
& \left\{\begin{array}{l}
\frac{1}{q_{h, k}} m_{h, k} \times \\
{\left[\rho_{a d s} R_{m}^{2} \frac{2 J_{1}\left(q_{h, k} R_{m}\right)}{q_{h, k} R_{m}} \exp \left[-\frac{\left(\sigma q_{h, k}\right)^{2}}{2}\right]\right.}
\end{array}\right. \\
& \lim _{q_{h, L} \rightarrow \infty} I\left(q_{h, k}\right)=C \sum_{h, k}\left\{\begin{array}{l}
-\rho_{\text {ads }} 2 \pi^{2} R_{v}^{2} R_{m}^{2} \times \\
2 \pi
\end{array}\right. \\
& \int_{0}^{2 \pi} d \delta^{\prime} \int_{0}^{\infty} d q_{r}{ }^{\prime} q_{r}{ }^{\prime} \frac{2 J_{1}\left(q_{r}{ }^{\prime} R_{m}\right)}{q_{r} R_{m}} \exp \left[-\frac{\left(\sigma q_{r}{ }^{2}\right)^{2}}{2}\right] \frac{2 J_{1}\left(\sqrt{\left.q_{h, k}{ }^{2}+q_{r}{ }^{\prime 2}-2 q_{h, k} q_{r}{ }^{\prime} \cos \left(\delta^{\prime}\right) R_{v}\right)}\right.}{\sqrt{q_{h, k}{ }^{2}+q_{r}{ }^{2}-2 q_{h, k} q_{r}{ }^{\prime} \cos \left(\delta^{\prime}\right) R_{v}}} \\
& {\left[-\rho_{\text {SBA-15 }} R_{m}^{2} \frac{2 J_{1}\left(q_{h, k} R_{m}\right)}{q_{h, k} R_{m}} \exp \left[-\frac{\left(\sigma q_{h, k}\right)^{2}}{2}\right]\right.}
\end{aligned}
$$


We define $C=C_{2}(\pi L)^{2}$, which is a constant independent of temperature if the amount of SBA15 seen by the neutron beam is unchanged. Equation (S10) is what we show as Equation (1) in the main context.

\section{Instrument Resolution and SANS Backgrounds}

Equation (S10) is a general equation which can apply to both empty SBA-15 and $\mathrm{CD}_{4} / \mathrm{SBA}-15$ if the length of the cylindrical mesopore can be treated as infinite.

To fit the experimental SANS data using the theoretical equations present above, the instrument resolution should be taken into account. The measured SANS intensity $I_{M}(q)$ can be expressed as:

$$
I_{M}(q)=\int \frac{I\left(q^{\prime}\right)}{\sqrt{2 \pi \delta(q)^{2}}} \exp \left[-\frac{\left(q-q_{m}\right)^{2}}{2 \delta(q)^{2}}\right] d q^{\prime}
$$

where $I\left(q^{\prime}\right)$ is the theoretical intensity calculated through equation (S10) with $q$ ' as a dummy variable. The instrument resolution is approximated to be a Gaussian function with the standard deviation $\delta(q)$ and $q$ dependent parameter $q_{m}$.

For each dataset of both empty SBA-15 and gas loaded SBA-15, Porod scattering $I_{p}(q)=c_{p} \times q^{-4}$ is added to account for the low $q$ upturn and Porod constant $c_{p}$ is also a fitting parameter. This low- $q$ scattering is due to the interface scattering from SLD contrast between the vacuum or $\mathrm{CD}_{4}$ vapor and the large powder particles in the sample. Here we briefly describe the validation of the addition of Porod scattering using empty SBA-15 as an example. This argument can be easily extended for $\mathrm{CD}_{4} / \mathrm{SBA}-15$ case. Let $B(\vec{r})$ as a uniform density distribution function covering the volume of big grain particle, that is, it defines the grain boundary of the hexagonally packed lattice. $P(\vec{r})$ is the density distribution of a cylindrical mesopore without gas loading and $L(\vec{r})$ is the distribution of hexagonally packed lattice. Therefore, the density distribution of the particle grain can be written as: $C(\vec{r})=\rho_{S B A-15} B(\vec{r})-\rho_{S B A-15} P(\vec{r}) \otimes(L(\vec{r}) \times B(\vec{r}))$, the notation $\otimes$

represents convolution. The scattering intensity can be written as $I(q)=\mid F[C(\vec{r})]^{2}$, where $F[]$ represents the Fourier transform. Therefore,

$$
\begin{aligned}
I(q) & =\rho_{S B A-15}{ }^{2}|F[B(\vec{r})]-F[P(\vec{r})] \times(F[L(\vec{r})] \otimes F[B(\vec{r})])|^{2} \\
& =\rho_{S B A-15}{ }^{2}\left\{|F[B(\vec{r})]|^{2}+|F[P(\vec{r})] \times(F[L(\vec{r})] \otimes F[B(\vec{r})])|^{2}-2 F[B(\vec{r})] \times F[P(\vec{r})] \times(F[L(\vec{r})] \otimes F[B(\vec{r})])\right\}
\end{aligned}
$$

The first term in the brace comes from the grain particle scattering and contributes to the Porod scattering. The second term is what represented in equation (S10) with $R_{v}=0$ and $\rho_{a d s}=0$ (the theoretical intensity of empty SBA-15), except that the instrument resolution is used for convolution instead of $F[B(\vec{r})]$ because the instrument resolution is dominant to determine the peak shape and intensity (see equation (S11)). Since $F[L(\vec{r})] \propto N$, where $N$ is the number of 
unit cells within the grain particle, the second term and third term in the brace are proportional to $N^{2}$ and $N$, respectively. $N$ is in general a big number and therefore the third term in the brace can be ignored compared with the second term. As a result, the Porod scattering (the first tem in the brace) can be directly added to theoretical scattering intensity we derive above.

For each dataset, a linear background is also added to the first order peak and the $q$ range below it, and a power law background is added to the second and third order peaks and $q$ range above them in order to account for the diffuse scattering of imperfect lattice order of hexagonally packed structure and effects from other defects. Different backgrounds are used because the baseline of scattering pattern changes with $q$ and temperature.

Figure S3 takes $\mathrm{CD}_{4} / \mathrm{SBA}-15$ at $225 \mathrm{~K}$ as an example and shows the experimental data, the best fits, Porod scattering, and the backgrounds added to the fitting. The theoretical intensity $I_{t h}$ calculated from equation (S10) is also shown and the resultant measured peak shape and peak intensity depend on the instrument resolution function described in equation (S11).

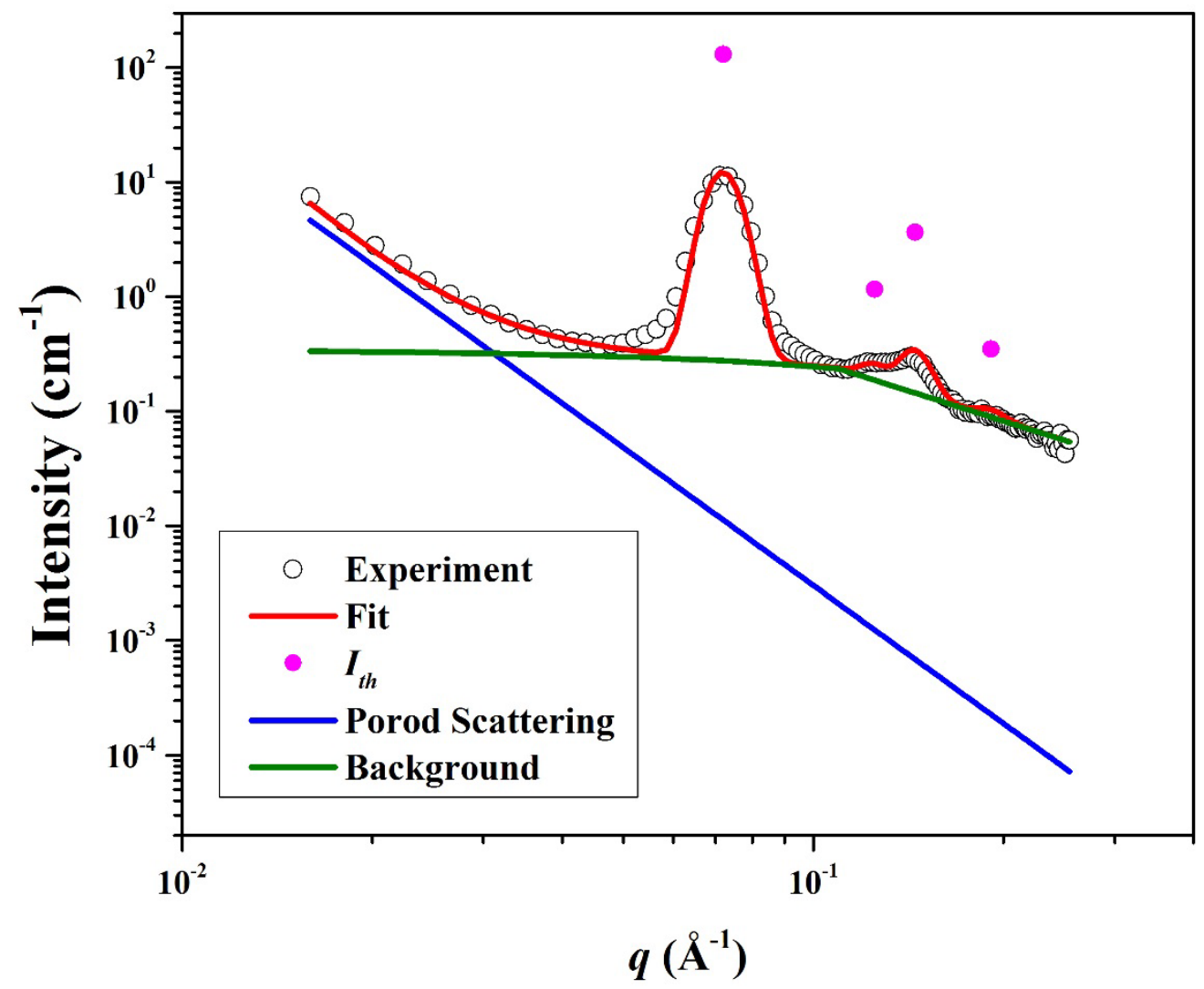

Figure S3. SANS experimental data (black circles), the fit (red line), the theoretical intensity $I_{\text {th }}$ calculated by using equation (1) in the main context (magenta circles), and the Porod scattering (blue curve) for SBA-15 loaded with $\mathrm{CD}_{4}$ at $225 \mathrm{~K}$ at pressure $\approx 100 \mathrm{kPA}$. A linear background (olive curve) is added to the first order peak region and the $q$ range below it and a power law background (olive curve) is added to the second and third order peaks and $q$ range above them. The nominal matrix radius $R_{m} 34.1 \pm 0.05 \AA$ and diffusive parameter $\sigma=7.5 \pm 0.09 \AA$ are fixed during the fitting. The fitted SLD of adsorbed $\mathrm{CD}_{4} \rho_{S L D, C D 4}$ and vapor core radius $R_{v}$ are $(6 \pm 1) \times 10^{10} \mathrm{~cm}^{-2}$ and $40 \pm$ $1 \AA$, respectively. Error bars of the experimental data represent one standard deviation and are smaller than the symbols. The confidence intervals for uncertainties of the fitting parameters also represent one standard deviation.

\section{Scattering Intensity of First Order Peak}


The SANS measurements for both cooling and heating scans are conducted to check the reversibility of the structure of $\mathrm{CD}_{4} / \mathrm{SBA}-15$ during gas adsorption. Figure S4 shows the SANS intensity of the first order peak of $\mathrm{CD}_{4} / \mathrm{SBA}-15$ for both cooling and heating scans. It is obvious that the intensity is reversible during the cooling and heating process except for the temperature region between $122 \mathrm{~K}$ and $122 \mathrm{~K}$ when capillary condensation happens. Although we do not have many data points for the heating scan during this temperature range, the inset in Figure S4 indicates that there is a hysteresis effect during capillary condensation. It should be noticed that the reversible SANS intensity at temperature below $112 \mathrm{~K}$ means that there is no bubble forming (partial filling) after capillary condensation takes place and therefore the structure for $\mathrm{CD}_{4} / \mathrm{SBA}$ 15 is reversible.

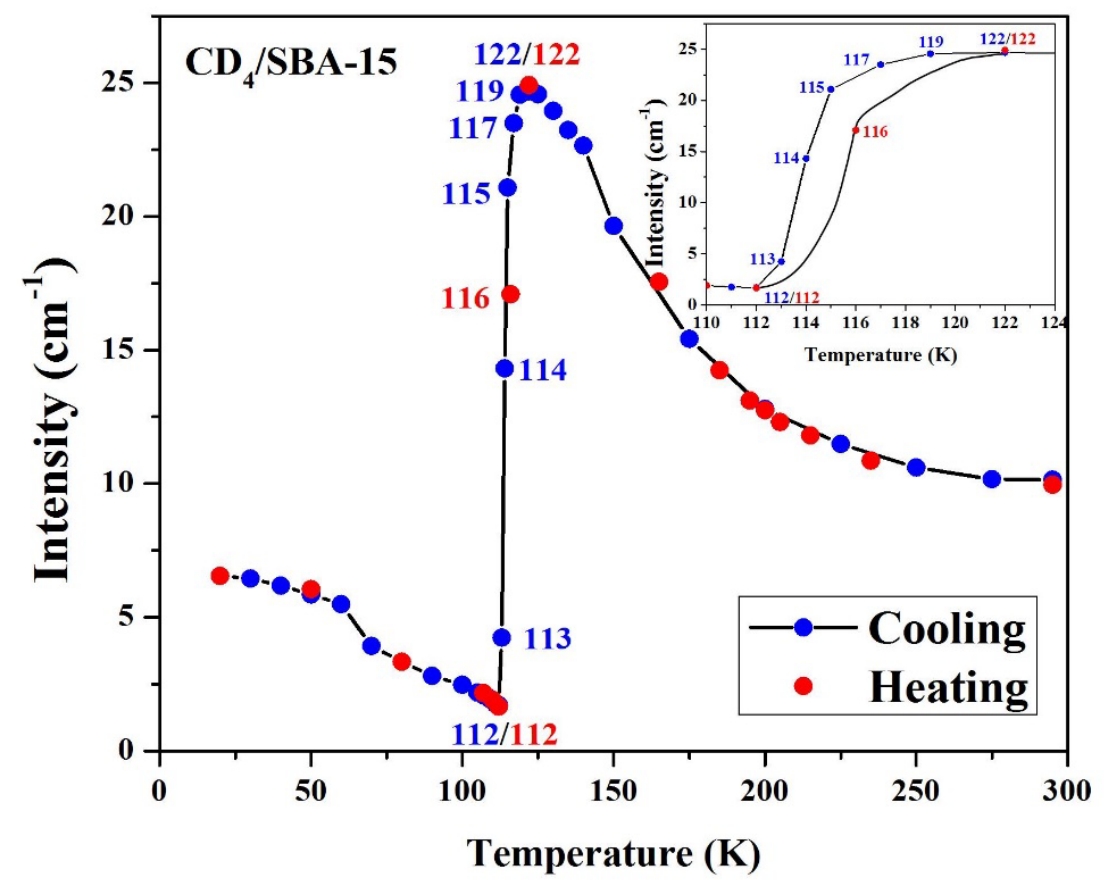

Figure S4. The first order peak intensity of SANS data during cooling (blue circles) and heating (red circles) for SBA-15 with $\mathrm{CD}_{4}$ loaded at pressure $\approx 100 \mathrm{kPa}$ as a function of temperature. The lines are only guides to the eye. The inset shows the enlargement of data taken during capillary condensation. Error bars represent one standard deviation and are smaller than the symbols.

SANS Fitting Results 
The parameters obtained from SANS fitting are listed in Table S1.

Table S1. SANS Fitting Parameters for $\mathrm{CD}_{4} / \mathrm{SBA}-15^{a}$

\begin{tabular}{|c|c|c|c|c|}
\hline $\begin{array}{c}\text { Temperature } \\
(\mathrm{K})\end{array}$ & $\begin{array}{c}C_{p} \\
\left(10^{35} \mathrm{~m}^{-5}\right)\end{array}$ & $\begin{array}{c}R_{v} \\
(\AA)\end{array}$ & $\begin{array}{c}\text { Mass Density } \\
\left(\mathrm{g} \bullet \mathrm{cm}^{-3}\right)\end{array}$ & $\begin{array}{c}\text { Total Adsorption } \\
\times 10^{15} \\
\left(\mathrm{~g} \bullet \mathrm{cm}^{-1}\right)\end{array}$ \\
\hline 295 & $3.45(3)$ & $45(13)$ & $0(1)$ & $3(18)$ \\
\hline 275 & $3.47(3)$ & $46(16)$ & $0(1)$ & $2(27)$ \\
\hline 250 & $3.63(3)$ & $42(4)$ & $0.4(3)$ & $6(7)$ \\
\hline 225 & $3.91(3)$ & $40(1)$ & $0.6(1)$ & $14(6)$ \\
\hline 200 & $4.32(4)$ & $40.2(8)$ & $1.0(1)$ & $24(5)$ \\
\hline 175 & $5.09(4)$ & $39.7(3)$ & $1.68(9)$ & $44(4)$ \\
\hline 150 & $5.93(5)$ & $39.2(1)$ & $2.56(5)$ & $75(4)$ \\
\hline 140 & $7.78(7)$ & $39.2(1)$ & $3.12(5)$ & $93(4)$ \\
\hline 135 & $6.84(7)$ & $38.90(8)$ & $3.10(4)$ & $98(3)$ \\
\hline 130 & $6.66(6)$ & $38.67(8)$ & $3.09(4)$ & $102(4)$ \\
\hline 125 & $7.54(7)$ & $38.31(7)$ & $3.03(3)$ & $106(3)$ \\
\hline 122 & $8.55(7)$ & $38.20(7)$ & $2.98(3)$ & $107(3)$ \\
\hline 119 & $12.43(9)$ & $37.83(7)$ & $2.77(3)$ & $107(3)$ \\
\hline 112 & $27.7(2)$ & 0.00 & $0.4789(3)$ & $183.2(6)$ \\
\hline 111 & $31.4(2)$ & 0.00 & $0.4850(3)$ & $185.6(6)$ \\
\hline 110 & $33.2(2)$ & 0.00 & $0.4933(3)$ & $188.7(6)$ \\
\hline 109 & $33.3(2)$ & 0.00 & $0.4950(3)$ & $189.4(6)$ \\
\hline 108 & $34.3(2)$ & 0.00 & $0.4989(3)$ & $190.9(6)$ \\
\hline 107 & $34.4(2)$ & 0.00 & $0.5002(3)$ & $191.4(6)$ \\
\hline 105 & $34.4(2)$ & 0.00 & $0.5044(3)$ & $193.0(6)$ \\
\hline 100 & $34.6(2)$ & 0.00 & $0.5147(3)$ & $196.9(7)$ \\
\hline 90 & $34.6(2)$ & 0.00 & $0.5262(4)$ & $201.3(7)$ \\
\hline 80 & $28.5(2)$ & 0.00 & $0.5381(4)$ & $205.9(7)$ \\
\hline 70 & $30.7(2)$ & 0.00 & $0.5454(4)$ & $208.7(7)$ \\
\hline 60 & $42.6(2)$ & 0.00 & $0.5686(5)$ & $217.6(7)$ \\
\hline 50 & $49.7(3)$ & 0.00 & $0.5716(5)$ & $218.7(7)$ \\
\hline 40 & $55.5(3)$ & 0.00 & $0.5700(5)$ & $218.1(7)$ \\
\hline 30 & $61.2(3)$ & 0.00 & $0.5703(5)$ & $218.2(7)$ \\
\hline 20 & $63.7(3)$ & 0.00 & $0.5705(3)$ & $218.3(7)$ \\
\hline
\end{tabular}

${ }^{a}$ In all the temperature, the structural parameters for the SBA-15 matrix are fixed as mesopore center-to-cetner distance $a=100.35 \AA$, SLD of the SBA-15 matrix $\rho_{S B A-15}$ is $(3.51 \pm 0.008) \times 10^{10} \mathrm{~cm}^{-2}$, nominal mesopore radius $R_{m}$ $=(34.1 \pm 0.05) \AA$, and diffusive parameter $\sigma=(7.5 \pm 0.09) \AA$. The confidence intervals for uncertainties represent one standard deviation.

\section{$\mathrm{CD}_{4}$ Pressure versus Temperature}


During the SANS measurement, $\mathrm{CD}_{4}$ pressure is monitored and maintained at about $100 \mathrm{kPa}$ by a $\mathrm{CD}_{4}$ gas cart at temperature above $112 \mathrm{~K}$. The change of $\mathrm{CD}_{4}$ pressure with temperature above $112 \mathrm{~K}$ is plotted in Figure S5. Below $112 \mathrm{~K}$, the valve connecting the $\mathrm{CD}_{4}$ gas cart and the sample cell is turned off to prevent the further condensation of gas in the bulk phase and the $\mathrm{CD}_{4}$ pressure inside the sample cell is not monitored.

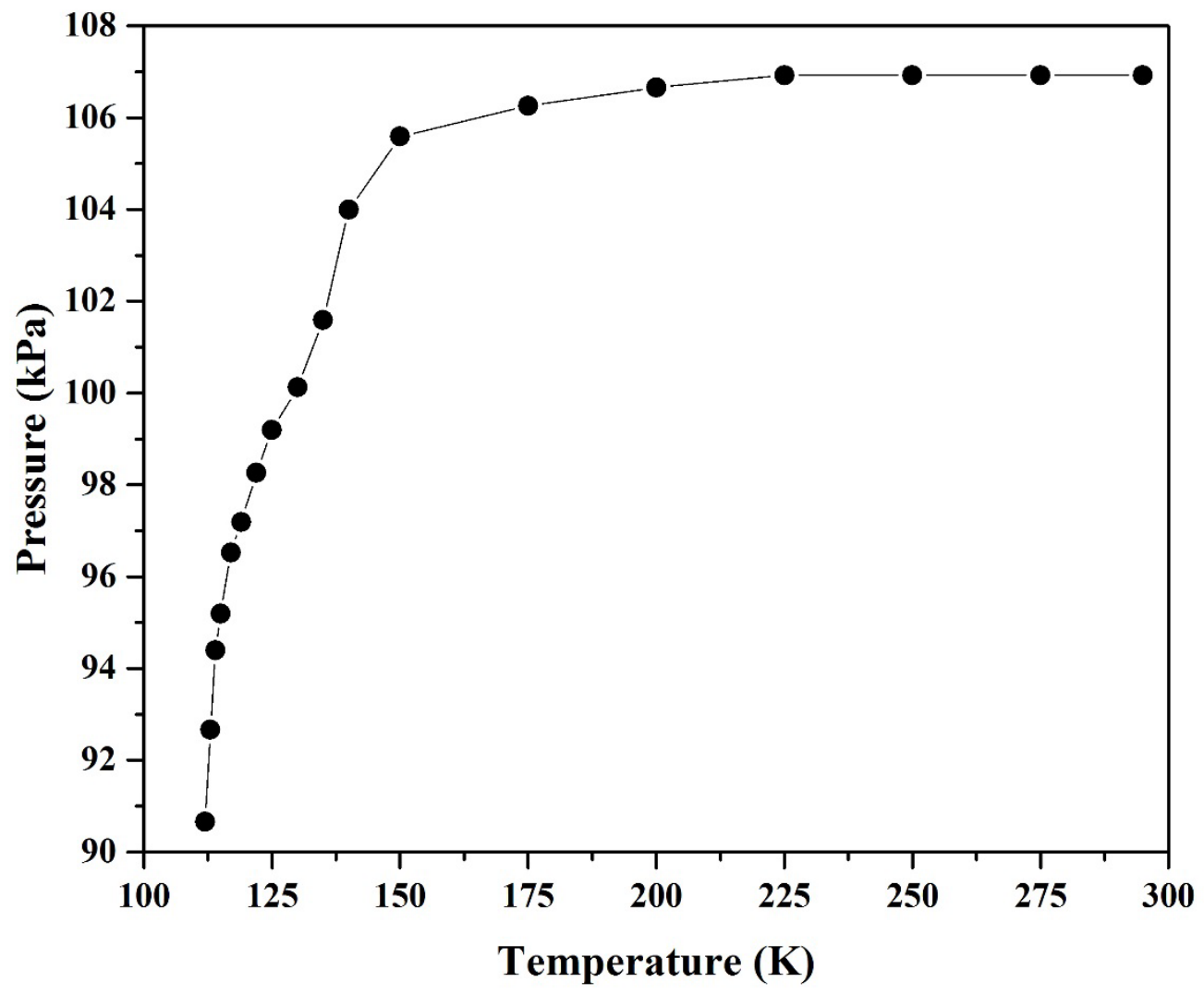

Figure S5. $\mathrm{CD}_{4}$ pressure versus temperature above $112 \mathrm{~K}$ during the SANS measurements.

\section{Calculation of Excess Adsorption}

When the amount of gas adsorption is small, i.e. at high temperature, the change of the scattering patterns is more sensitive to the excess adsorption, $\varepsilon_{a d s}$, than to $R_{v}$ and $\rho_{a d s}$. This can be demonstrated easily by using the simplest core-shell cylinder case when both $I n_{m \text {-ads }}$ and $I n_{\text {ads-v }}$ are sharp interfaces. The form factor of simple core-shell cylinder with its axis perpendicular to the wave vector $q$ and with negligible vapor core SLD $\left(\rho_{v}=0\right)$ can be expressed as: 


$$
\begin{aligned}
& P(q)=\left|\left[\left(\rho_{a d s}-\rho_{\text {SBA-15 }}\right) V_{m} \frac{2 J_{1}\left(q R_{m}\right)}{q R_{m}}-\rho_{a d s} V_{v} \frac{2 J_{1}\left(q R_{v}\right)}{q R_{v}}\right]\right|^{2} \\
& =\left.(\pi \mathrm{L})^{2}\left[\rho_{a d s} R_{m}^{2} \frac{2 J_{1}\left(q R_{m}\right)}{q R_{m}}-\rho_{a d s} R_{v}^{2} \frac{2 J_{1}\left(q R_{v}\right)}{q R_{v}}-\rho_{\text {SBA-15 }} R_{m}^{2} \frac{2 J_{1}\left(q R_{m}\right)}{q R_{m}}\right]\right|^{2}
\end{aligned}
$$

Let $f(R)=R^{2} \frac{2 J_{1}(q R)}{q R}$, then $\rho_{S B A-15} R_{m}^{2} \frac{2 J_{1}\left(q R_{m}\right)}{q R_{m}}=\rho_{S B A-15} f\left(R_{m}\right)$ is the contribution from the SBA-15 solid matrix and is independent of the gas adsorption. When the amount of adsorption is small, $\Delta R=R_{m}-R_{v}$ is small enough to do the Tayler expansion and $\varepsilon_{a d s}=2 \pi R_{m}\left(\rho_{a d s} \Delta R\right)$. Equation (S14) reduces to:

$$
\lim _{R_{m} \rightarrow R_{v}} P(q)=(\pi L)^{2} \mid\left[\rho_{a d s} \Delta R f^{\prime}\left(R_{m}\right)-\rho_{S B A-15} f\left(R_{m}\right)\right]^{2} \approx(\pi L)^{2}\left[\left[\varepsilon_{a d s} f^{\prime}\left(R_{m}\right) /\left(2 \pi R_{m}\right)-\rho_{S B A-15} f\left(R_{m}\right)\right]^{2}\right.
$$

Therefore, $\varepsilon_{a d s}$ is the characteristic parameter that determines the scattering pattern change when adsorption amount is small. Hence, when fitting the data using $R_{v}$ and $\rho_{a d s}$, these two parameters are actually strongly correlated when $\varepsilon_{a d s}$ is small. This proof can be further extended to the case with a diffuse layer for the empty matrix. Of course, when the temperature is close to $T_{c}$, the amount of adsorption is so large that keeping only the first order term of the Taylor expansion is not accurate enough.

For the cases of cylindrical pore with diffuse $I n_{m \text {-ads }}$ interface, we have calculated the scattering patterns. With given $\varepsilon_{a d s}$, there are many combinations of $R_{v}$ and $\rho_{a d s}$. We systematically vary $R_{v}$ and calculate the intensity change of the $1^{\text {st }}$ peak in the scattering patterns after convoluting with the instrument resolution functions. Figure S6a and Figure S6b show the calculated $1^{\text {st }}$ peak intensity for Gaussian model (equation (1) in the main context and equation (S10) in SI) and linear model (model (4) in the main context), respectively, as a function of core radius $R_{v}$ at different values of $\varepsilon_{\text {ads }}$. For both models, the peak intensity is independent of $R_{v}$ at low $\varepsilon_{\text {ads }}$. Therefore, if the only prominent peak is the $1^{\text {st }}$ order peak, its intensity will be solely determined by $\varepsilon_{a d s}$ when $\varepsilon_{a d s}$ is small. When $\varepsilon_{a d s}$ becomes large, the relative intensity becomes more sensitive to the change of $R_{v}$. And $R_{v}$ and $\rho_{a d s}$ become more independent to each other. 
(a)
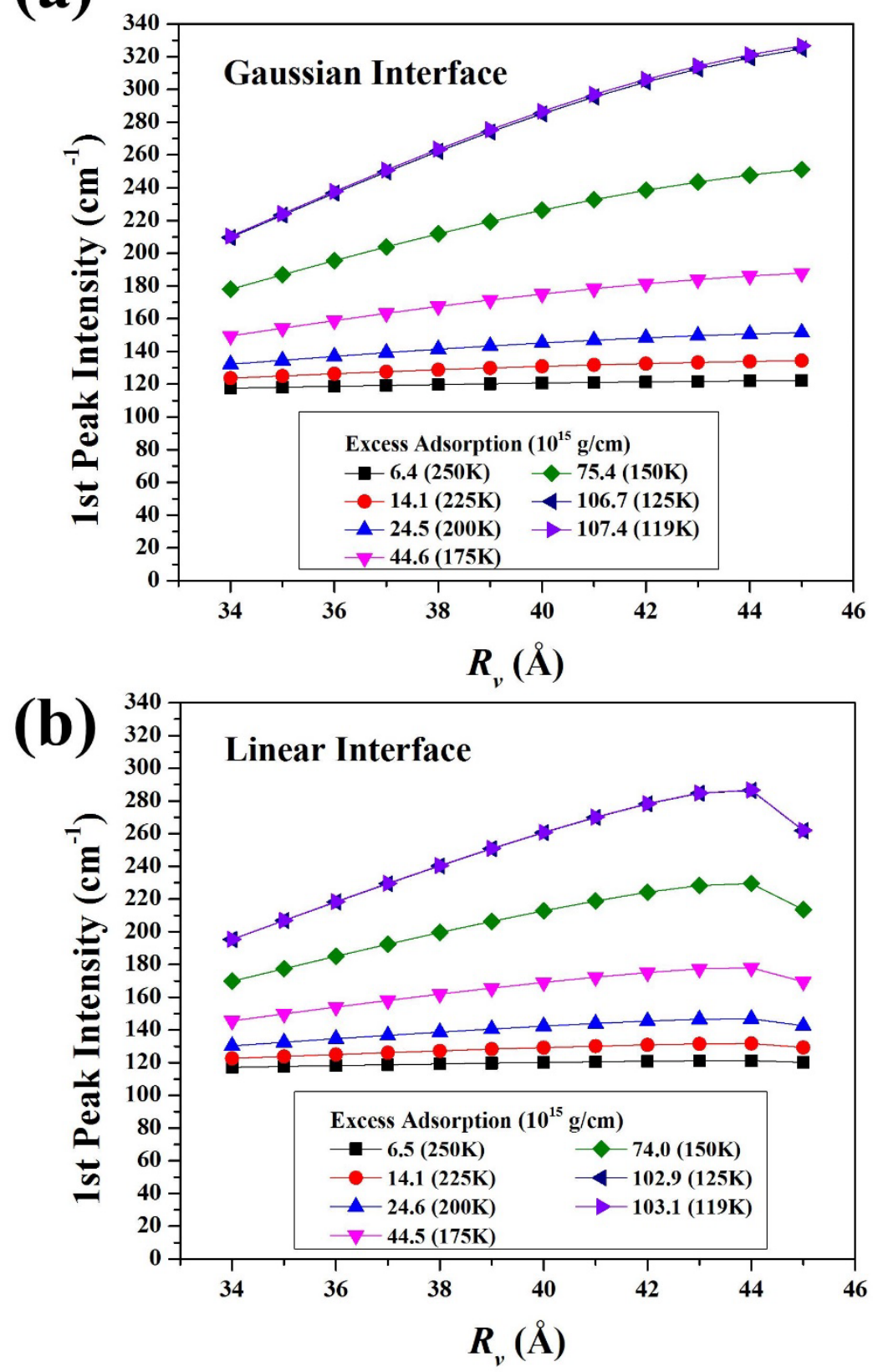

Figure S6. Simulated SANS $1^{\text {st }}$ order peak intensity as a function of vapor core radius $R_{v}$ when $\varepsilon_{a d s}$ is fixed for (a) the Gaussian diffuse interface model and (b) the linear diffuse interface model. The index indicates the $\varepsilon_{a d s}$ value in unit of $10^{15} \mathrm{~g} / \mathrm{cm}$, which corresponds to the value obtained from fitting the SANS data for $\mathrm{CD}_{4} / \mathrm{SBA}-15$ at temperature shown in the parentheses. 


\section{$N_{2}$ Adsorption Isotherm}

The adsorption isotherm for $\mathrm{N}_{2}$ into the synthesized SBA-15 is plotted in Figure S7. The textural properties obtained from $\mathrm{N}_{2}$ isotherm are summarized in Table S2. Surface area measurement was performed by means of a Beckman Coulter SA 3100 Surface Area Analyzer ${ }^{\mathrm{S} 1}$ after outgassing the sample in vacuum condition at $200{ }^{\circ} \mathrm{C}$ until the pressure of $4.0 \mathrm{mmHg}$ was reached. The specific surface area was calculated with the Brunauer-Emmett-Teller (BET) method, and the size distribution and diameter were obtained by applying the Barrett-JoynerHalenda (BJH) method to the desorption branch of the isotherm.

The ratio of $\mathrm{N}_{2}$ adsorption amount when all the surfaces are fully covered prior to capillary condensation $\left(282.73 \mathrm{cc} / \mathrm{g}(\mathrm{STP})\right.$ at $\left.\mathrm{P} / \mathrm{P}_{0} \sim 0.51\right)$ to adsorption amount when all pores are fully filled right after capillary condensation (499.53 cc/g (STP) at $\mathrm{P} / \mathrm{P}_{0} \sim 0.73$ ) is 0.57 . This is consistent with the results we extract from SANS data analysis, where the absolute adsorption, $M_{a d s}$, prior to and after the capillary condensation has the ratio $M_{a d s}(T=119 \mathrm{~K}) / M_{a d s}(T=112 \mathrm{~K})$ $=0.58$.

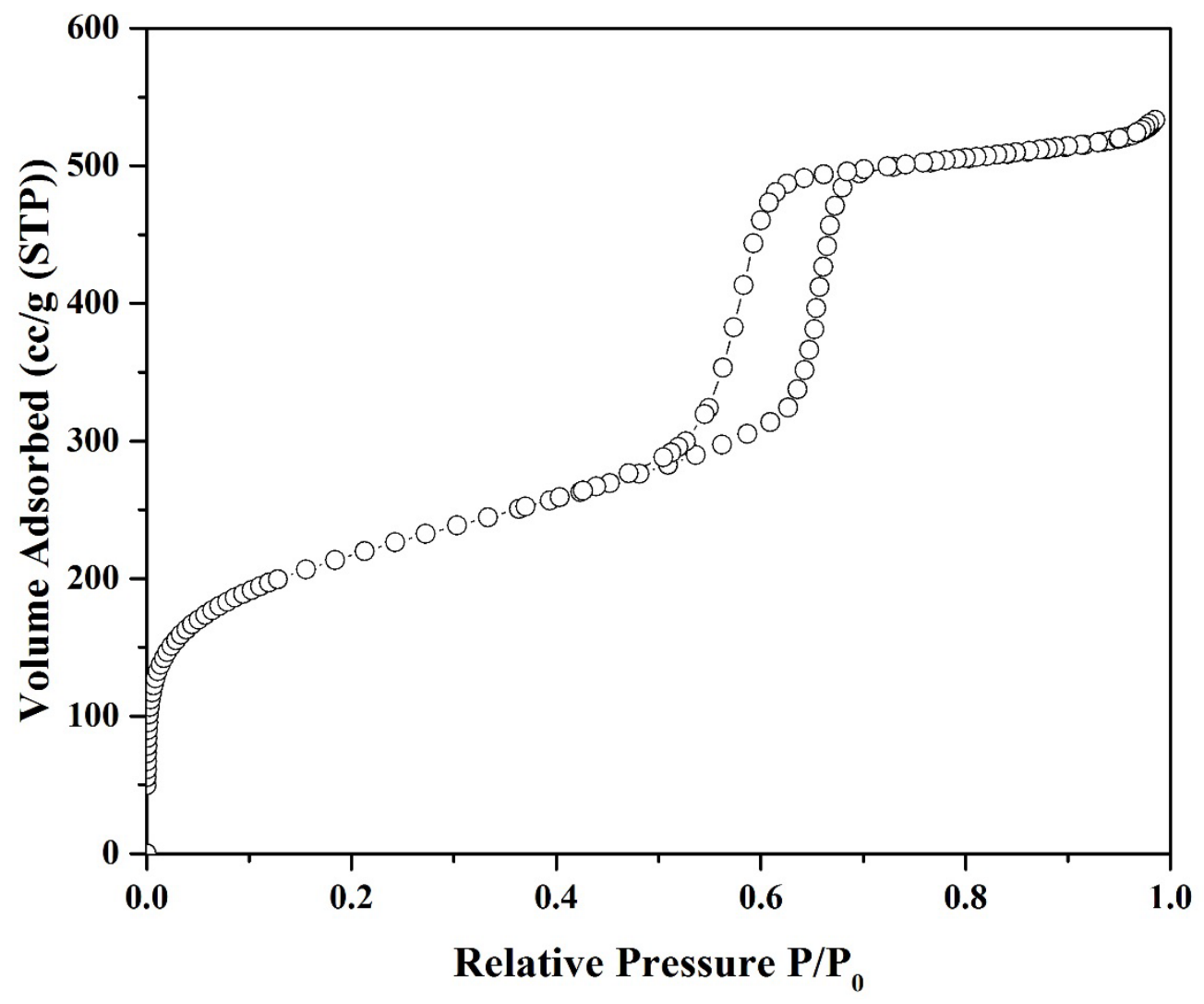

Figure S7. Adsorption isotherm measurement for $\mathrm{N}_{2}$ onto SBA-15. 
Table S2. Textural Properties obtained from $\mathrm{N}_{2}$ Isotherm

\begin{tabular}{|c|c|c|}
\hline $\begin{array}{c}\text { BET surface area } \\
\mathrm{m}^{2} / \mathrm{g}\end{array}$ & $\begin{array}{c}\text { pore size } \\
\mathrm{nm}\end{array}$ & $\begin{array}{c}\text { pore volume } \\
\mathrm{ml} / \mathrm{g}\end{array}$ \\
\hline 781.15 & 47.6 & 0.8198 \\
\hline \multicolumn{3}{|c|}{} \\
\hline
\end{tabular}

\section{Reference}

(S1) Identification of a Commercial Product Does Not Imply Recommendation or Endorsement by the National Institute of Standards and Technology, nor Does It Imply That the Product Is Necessarily the Best for the Stated Purpose. 